Il semble bien qu'en présence de semblables résultats à attendre, les Administrations compétentes soient disposées, et nous sommes heureux de le constater, à faire tombrr les obstacles qui s'opposent encore à leur aménagement et à l'utilisation rapide de notre richesse hydraulique. Déjà, pour les forces hydrauliques du domaine privé, les initiatives individuelles ont eu pour conséquence la transformation économique de régions pauvres. Cerles, en ce qui concerne le domaine public, on conçoit qu'en présence des exigences de l'heure préscnte, l'Etal ait le légitime souci de ne pas se dessaisir a tout jamais de ce domaine, et l'industrie s'est inclinée devant les réserves qu'il a pu convenir de formuler a cet égard. Tout démontre pourtant que la collectivité ne peut manquer de recueillir une large participation dans les avantages à obtenir et le Parlement idevra, par une appréciation équitable de la situation, établir un récime libéral. Les bases d'une cntente loyale sont possibles. Il nous paraît nécessaire de les trouver avant que d'autres pays prennent sur nous l'avance que nous avous su leur disputer.

In travaillant à ce résultat, nous aurons rempli, mes chers Collègues, l'un des buts qui nous sont assignés par ceux qui ont tracé notre voie: poursuivre l'application la plus chlondue des forces et des richesses du pays.

Nos lecteurs ont retrouvé, dans le discours précédent, la documenlation que nous avons déjà si souvent mise sous leurs yeux. Mais, en la circonstance, elle emprunte à la haute situation de l'auteur si qualifié pour traiter ce sujet, une valeur particulière ; d'ailleurs, l'exposé qu'on vient do lire du développement de l'Electrochimie comporte une rue d'cnscmble d'où se dégagent d'une manière saisissante les perspeclives d'avenir de cette industie : c'est à ce double litre que nous avons reproduit ces pages.

En les lisant,il n'est pas un technicien qui ne remarquera la modestie de M. Gal, parlant de tous, sauf de lui à qui la science électrochimique cst pourtant redevable de travaux si féconds. Ceci prouve bien une fois de plus que c'est à leur modestie que se reconnaissent les vrais savants.

\title{
AMÉNAGEMENT D'UNE HAUTE GHUTE EN HAUTE MONTAGNE
}

D'unc intéressante conférence faite réccmment à la Société d'Agriculture, Sciences et Industrie de Lyon, par notre collaboraleur M. Charles, nous extrayons la description suivante. Elle montre bien de quelle somme d'audace, do science of d'énergie les mineurs de la houille blanche doivont faire preuve pour conquérir les merveilleuses et inépuisables richesses que la nature a placées aux flancs do nos monlagnes.

Une chute, quelle que soit sa puissance, n'a beaucoup do valeur que si ellc est à peu près constante ou, du moins; si sa force minima est encore assez grande et de durée assez combe pour que le secours demandé à tout autre source d'énergie soit limité et n'entraine pas l'immobilisation de capilaux considérables.

Cefle condition n'est réalisée que lorsquion peut accumuler en un réservoir suffisamment grand l'eau inutilisée pen-

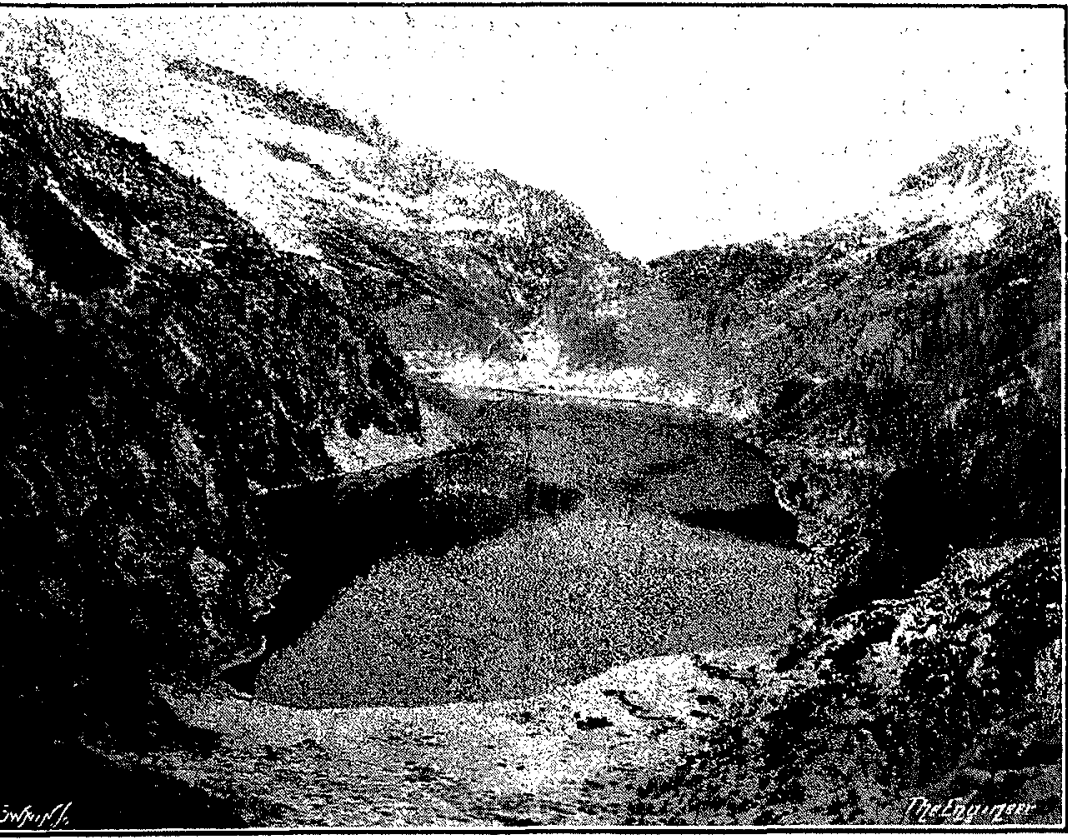

dant une partie de la journée ou de l'amnéc, pour en lirer parti aux heures où la demande de force est plus grande, ef pendant les périodes où le froid et la sécherosse réduisent le débit d'u cours d'eau exploité.

Plus la hauteur de chute utilisée est grande, plus pelil est le. volume d'eau nécessaire pour la production d'une force donnée, et plus faible, par conséquent, le volume de la réserve à constituer pour les moments de pénurie.

De plus, une installation hydroélectrique puissante en basse chute nécessite des machines énormes, landis qu'en haute chute ces mêmes éléments, sauf la longueur do la conduite forcée, se réduisent en dimension, en poids et en prix. Aussi, cherchc-t-on de préférence les hautes chutes alimentées par des ruisseaux provenant de glaciers et traversant des lacs ou des vallées que l'on peut, à l'aide de travaux relativement peu couteux, transformer en réscrvoirs assez vastes pour parer, dans une large mosure à l'irrégularité de débit de ces ruisseaux.

L'installation hydroélectrique de l'Adamello nous fournira un exemple typique des travaux de ce genre.

\section{INSTALLATION HYDROÉLECTRIQUE DE L'ADAMELLO}

L'Adamello s'élève à plus de 3500 mètres sur un des contreforts des Alpes Réthiques qui descendent vers la plaine lombarde, près de Brescia.

L'écoulement du glacier vers l'ouesl, forme un réscau de torrents qui se précipitent écumants entre des sommets converts, jusqu'à la limite des neigos, de pins el do hêtres, ot descendent par une succession de vallées sauvages se jeter dans I'Oglio au village de Cedegolo.

En ce point, cette rivière reçoit la Poglia, alimentéc ellcmême par l'Adaniè, le Salarno et la Poglia d'Arno, śmissairc du lac d'Arno.

Le lac d'Arno, situć à I 800 mètres d'allitudes, a 57 r 000 mètres carrés. Un ingénieur, $M$. Luigi Stucchi, frappé de la valeur industrielle de ce réservoir nalurel presque inaccessible, en fit l'acquisition après s'êtro assuré Ja concession des affluents de la Poglia ; il transmit ses droits à la Société Générale Electrique de l'Adamello, formée en avril rgo7 pour utiliser les forces hydrauliques du val Camonica. Trriz 
semaines après, on commençait l'élaboration des plans et, dès xaro, les deux stations d'Isola et de Cedegolo étaient à même de remplir les termes de leurs contrats pour Milan et la province de Brescia.

Une telle rapidilé d'exécution est extrêmoment remarquable parce que, indépendamment des procès et des expertises que soulevèrent les barreurs de chintes pour avoir des indemnités, les conditions physiques de l'entreprise furent extrêmement rigoureuses.

La demande de force n'élanı pas constante, le lac fournit pendant les heures où le besoin s'en fait sentir, la quantité d'eau nécessaire et retient ensuite l'eau des nuisseaux pour reconstituer la réserve journalière. Il sert également à former une réserve pour les périodes de basses eaux.

Pour améliorer les conditions d'exploitation on augmenta beaucoup la capacilé du lac et l'on y conduisit les eaux de l'Adamè, tributaire d'un bassin différent. de l'exécution des plans, mais encore de la mise en train et du fonctionnement des services d'entretien et d'approvisionnement de l'entreprise. Tâche compliquée, car elle cormprenait non seulement l'abri, le couchage, la nourriture et le service de santé, mais aussi le service des communications entre la plaine, les bâtiments en construction à Cedegolo et à Isola, le lac d'Amo et les groupes d'ouvriers disséminés tout le long de l'ouvrage.

Ils établirent 20 kilomètres de voie Decauville, desservis par des locomotives à essence, étudićes spécialcment pour cette entreprisc, et qui donnèrent toute satisfaction.

Il fallut tout d'abord construire une station ccntrale provisoire sur une dérivation du Salarno. On y. plaça deux groupes hydroélectriques de $x 50$ chevaux chacin. Le courant, produit sous une tension de 3 ooo volts, était distribué dans les chantiers où des transformateurs l'abaissaient ì 220 volls. Il scrvail ì actionner les treuils, les grues et les

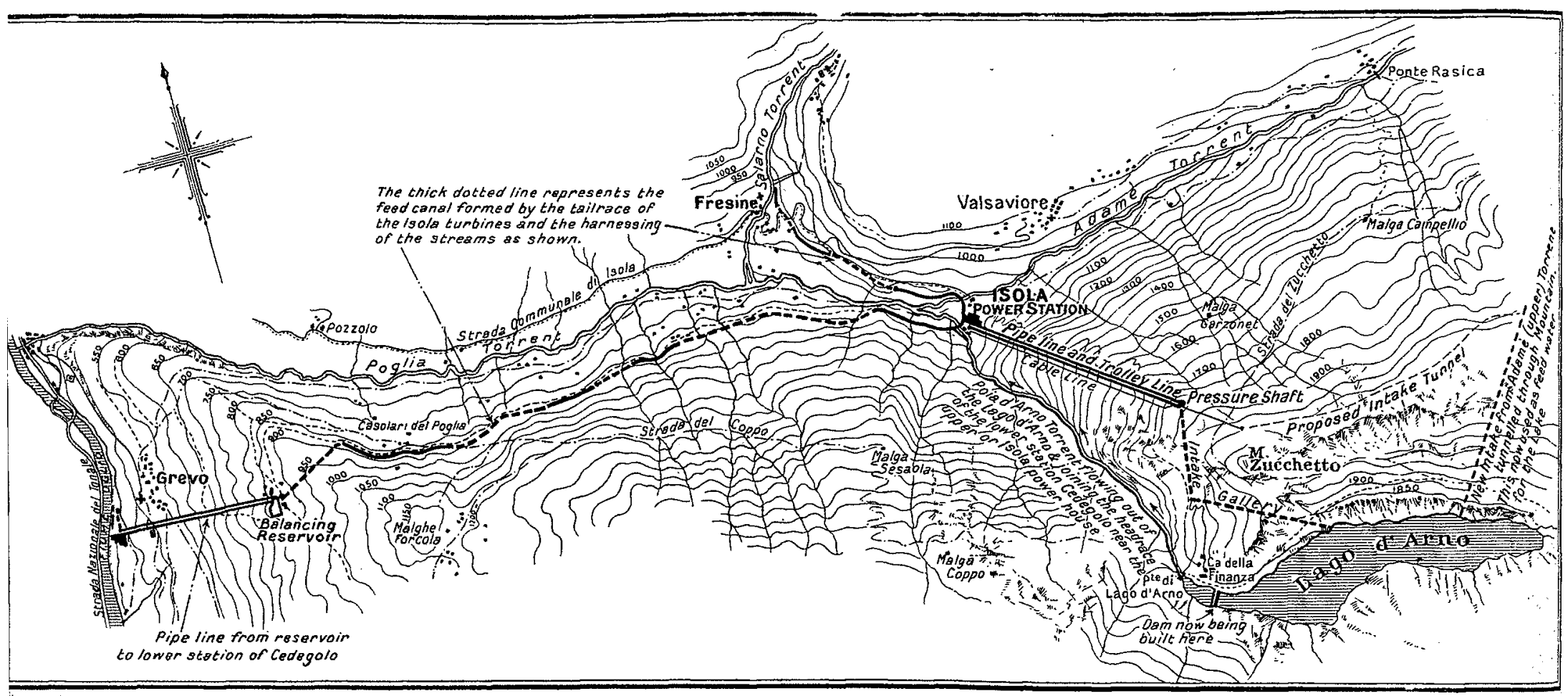

L'eau du lac alimente l'usine d'Isola ; le canal de fuite do cette dernière reçoit les eaux de sources et les eaux superficielles qui lui sont amenées par l'Adamè, le Salarno et la Poglia d'Arno, et alimente à son tour une seconde usine, celle de Gedegolo. Celle-ci, siluéc à 5 kilomètres de l'usine d'Isola, sur la rive de l'Oglio, constitue la station centrale; clle reçoit la force produite à l'usine d'Isola et l'envoie sous une tension de $7^{2}$ ooo volts concurremment avec celle qu'elle engendre elle-même à la station de San-Giovanni, près de Milan, où elle est distribuée.

Le profil ci-après (page 5o) donne immédiatement l'impression de l'économie du projet.

Organisation du cuantier. - Il est difficile de donner une idée cxacte, à ceux qui n'ont pas visité la partie supérieure de la chute, de la lutte engagée contre les forces de la nature par des groupes d'hommes isolés les uns des autres et du reste du monde sur ces montagnes sauvages.

Les travailleurs, environ r.900 hommes, furent divisés en groupes commandés par des contremaîtres et un chef de chantier, placés cux-mêmes sous les ordres des ingénieurs, MM. Gaetano Carinati et Carlo Vassena.

Ces Messieurs furent chargés non seulement de l'étude et chariots pour les transports, les pompes d'épuisement et les compresseurs d'air pour les travaux souterrains, les moteurs de 6 lignes à traction funiculaire et de deux câbles porteurs aériens ; enfin, les services d'éclairage el de communication par télégraphe et téléphone. Trente-doux postes téléphoniques reliaient les divers chantiers entre eux et au bureau central. Le service électrique était dirigé par l'ingénieur M. Roncaldier.

La direction générale de l'entreprise fut confiée à M. Adolfo Covi, ingénieur à Milan, dont le nom, lié au succès des installations de Paderno et Trezzo, était par lui-même un garant du résultat final.

Telles étaient les dispositions prises quand on attaqua les travaux simultanément à Cedegolo, à Isola et au lac d'Arno.

Exécution du travail. - Les berges du lac d'Arno sont extrêmement raides, la profondeur de l'eau atteint immédiatement 25 mètres contre les bords el l'on relève 60 mètres de fond a centre.

On calcula qu'on pourrait disposer d'une réserve de 12 millions de mètres cubes d'eau en pratiquant un exutoire au lac à 25 mètres au-dessous de la surface normale du lac; en ce point la superficie de la nappe est de 37 ooo mètres 
carrés, alors qu'à la surface du lac elle est, comme on l'a dit, de 5jlooo mètres. On s'assura que la précipitation annuelle est bien supérieure aux I ooo millimètres nécessaires pour remplacer le volume prélevé.

Dans les conditions normales on pouvait ainsi dériver 2 ooo litres par seconde pendant huit heures par jour et produire à Isola 19 ooo chevaux. Ce ri'était pas suffisant. On se décida à transformer le lac en un immense réservoir. Son bassin est de 14 kilomètres carrés seulement, mais on escomptait la présence de sources souterraines et on s'appuyait sur le fait que même, alors que lc niveau était le plus bas, il s'écoulail encore 3 oo litres par seconde. Pour recueillir ce trop-plein et augmenter la capacité du lac, on décida de barrer la gorge rocheuse de l'émissaire et, en même temps, de capter l'ean de l'Adamè pour la conduire dans le lac. On reconnut qu'on pouvait relever sans danger le nireau du lac de $25 \mathrm{~m}$., porter sa capacité utile de r 2 millions à 29 millions de mètres cubes et augmenter le débit utilisable ì Isola de 2000 à 4800 litres par seconde.

C'est dans cel esprit que l'on commença les travaux.

Tunnes de prise j'eau. Galemie sols pression. - Cetté galerie devait déboucher à 25 mètres au-dessous du niveau de l'eau. Pour alteindre ce point à travers la montagne, on décida de percer une galerie coudée prenant jour au fond de 3 crevasses profondes.

On partagea la longueur totale de $x .465$ mètres en trois sections : la première, de 800 mètres, allant de la paroi du lac à la première "fenêtre "; la seconde, de 200 mètres, allant de la première à la seconde fenêtre à travers la deuxième crête ; la troisième, d'environ 465 mètres, allant de la deuxième fenêtre au point choisi comme sortie de la galerie de prise d'eau et point de départ de la conduite forcée.

Dans les deux dernières sections, on ne rencontra aucune difficulté autre que celles inhérentes à la perforation des tunnels en roche dure. La première section, par contre, ménagea toutes les surprises désagréables possibles.

On avait projeté de percer cette partie de la galerie d'abord horizontalement sur 300 mètres, à partir d'u lac, et de lui donner une pente de r pour roo sur les 600 mètres suivants. On devait l'altaquer simultanément par les deux extrémités, en laissant entre la paroi du lac el l'entréc de la galerie un mur de roche que l'on abattrait une fois le reste de la galerie achevé.

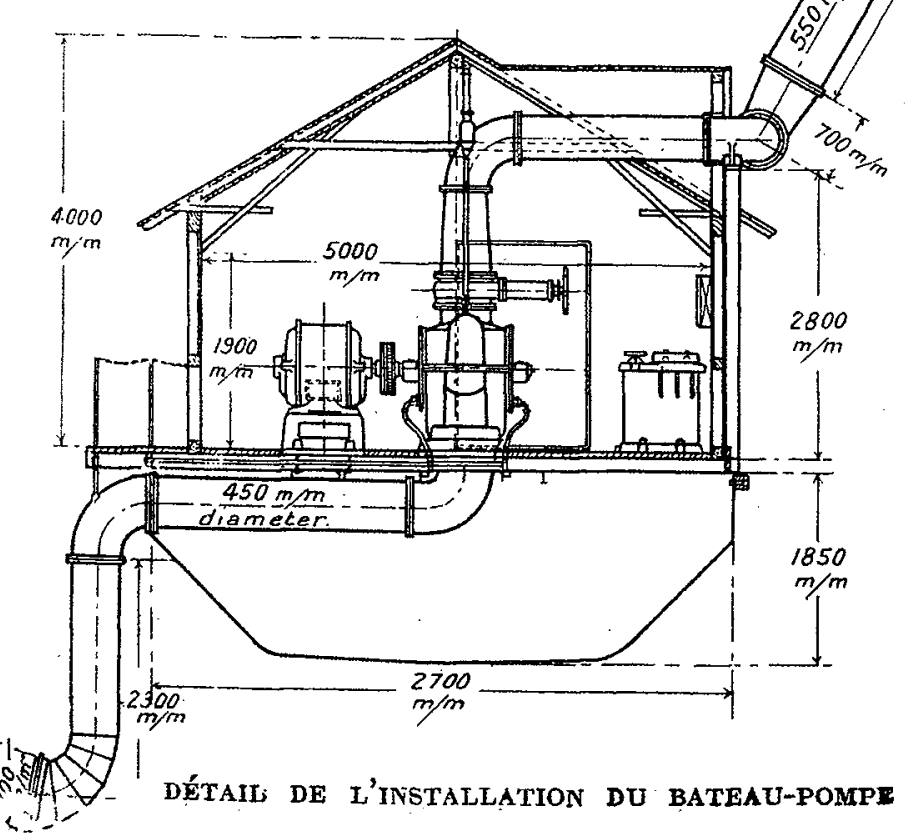

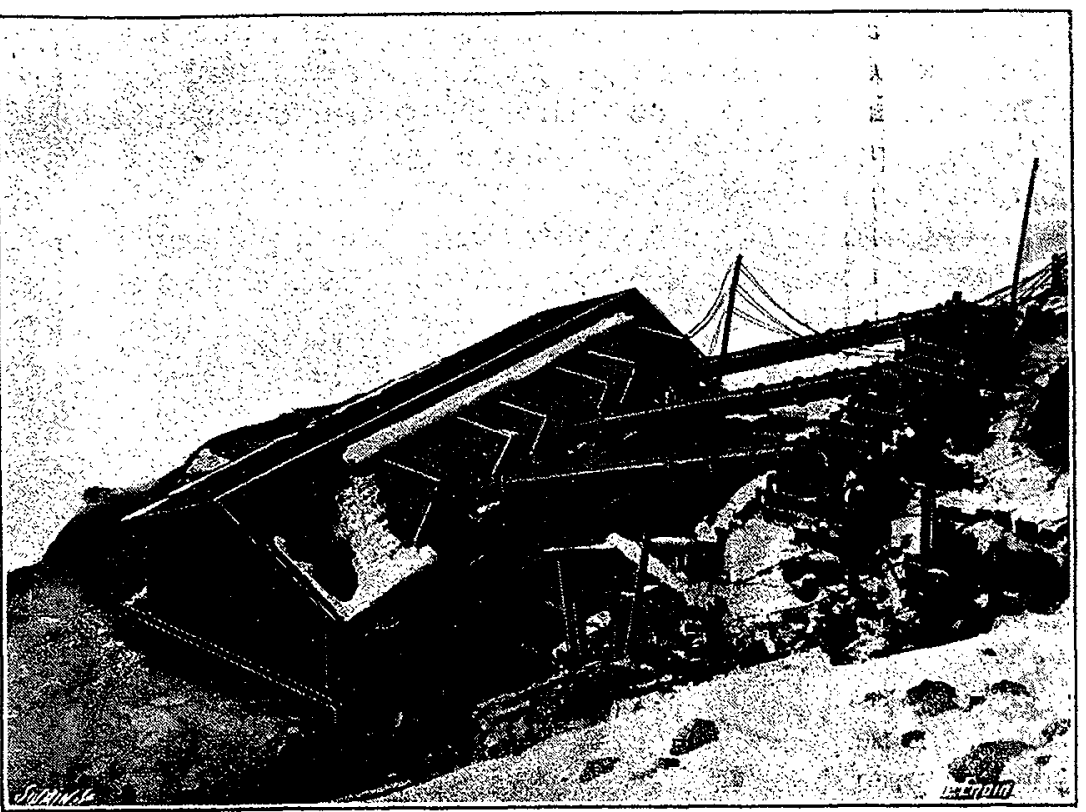

VUE "PATTIELLE DU BATEAU-POMPE EN SERVICE.

Suivant ces dispositions, on entama an niveau de l'eau une galerie horizontale de i 5 mètres de longueur et, comme on supposait n'avoir à aredouter aucune infiltration, on commença à creuser le puils vertical qui devait, à la profondeur de 25 mètres, atteindre le niveau de la prise d'eau. Mais dès qu'on eut creusé quelques mètres, les premières infiltrations apparurent. Les infiltralions s'étant également manifestées à l'autre extrémilé de la galerie, on ne chercha pas à prolonger plus loin la galerie d'attaque. Le travail de fonçage, à l'aide des perforatrices à air compximé, fut poursuivi devant des difficultés croissantes de jour en jour, augmentées par le peu d'espace dont on disposait. A chaque volée, il fallait retirer les pompes électriques, puis. les redescendre.

Après un total de onze mois de ce travaii dangereux, exécuté jour et nuit el portanl sur plus de deux années en raison des conditions climatériques, on at . génieurs et aux grosses gratifications allouées aux ouvriers. A ce point, on dut abandonner le travail de forage du puits, car les pompes, travaillant par deux et même. par trois, ne pouvaient plus faire face à la venue d'eau.

On gardait l'espoir de percer les 6,5o derniers mètres de bas en haut, à partir de la galcrie de prise d'eau.

Dans celle-ci, on avait, dès le début, rencontré des infiltrations ; elles avaient augmenté à chaque mètre d'avancement el los ouvricrs travaillaient avec de l'eau presque glacéc. i 4 degrés jusqu'aux genoux. La pente trop faible rendait impossible l'épuisement de la galerie par des pompes. La roche, très dure, nécessitait l'emploi d'explosifs puissants, que la tempéralure, très basse, rendait dangereux à manipulcr. Après quelrues accidents séricux, suivis de mort d'hommes, les directcur's furent obligés de renvoyer plusicurs livraisons de dynamite el de faire usage d'explosifs insensibles au froid, mais moins puissants. Malgré ces précautions et les salaires allćchants offerts, les ouvriers se retiraient l'un après l'autre et le moment vint où les ingénieurs fur'ent abandonnés à leurs seuls efforts. Ce furent eux qui percèrent les derniers mètres de rochers pour arriver au puits vertical. La jonclion, qui présentait les plus grands dangers, fut accomplie sous un torrent d'eau le 24 novembre igog.

La galerie sous pression fut enduite de béton et façonnée en tunnel cylindrique de $\mathrm{I}^{\mathrm{m}} 8 \mathrm{o}$ de diamètre. 
Vanne d'entrée. - Dans le puits, on installa une vanne de $1,60 \times 1,60$ équilibrée de façon à être manouvrée aisément sous la charge de 50 mètres de hauteur d'eau qu'elle aurait à supporter une fois le plan d'eau du lac relevé à la hauteur jugée nécessaire.

La jonction de la galerie horizonlale avec le puits au niveau désiré constituait une importante victoire. L'étape suivante consistail à percer, dans le prolongement de la première, une galerie horizontale, aussi loin que possible, et à tenter de faire sauter la cloison la séparant du lac. Les infiltrations furent telles, qu'on ne put aller audelà de x3 mètres. En ce point, $o n$ creusa une chambre de mine en forme de $\mathrm{Z}$ renversé à gauche du lunnel. On ne pouvait avoir une idée précise de la dislance exacte qui séparait les travailleurs du lac, car on ne pouvait employer des scaphandriers et les sondages n' ind iq'u aient que le contour du talus et non la roche cachéc sous les débris accumulés pendant des siècles.

On décida de donner à la charge un rayon d'action de 20 mètres et, pour cela, la chambre fut garnie de deux tonnes de gélaline à $9^{3}$ pour roo de nitroglycérine. Tout le zigzag ful garni de maconnerie de ciment et, par mesure de précaution, lc puits ot la galerie furent cn outre remplis d'eau au moyen d'un siphon qui puisail dans le lac.

Ces préparalifs prirent deux mois el, lc 2 févier igro, la charge ful enllammée avec un courant à 3.000 volts.

le son de la délonalion donna inslantunémeni aux mincurs l'impression d'un " raté ". Un moment après, les neiges tombèrent en avalanches des montagnes voisines, landis que des lomnes d'eau, mêlées de maçonneries, de charpentes, de machineric cl de tuyauteric étaient vomies par la galcrie supérieure et allaient tomber au loin dans le lac.

Le gaz, trouvant une fissure dans la roche, avaient suivi la faille sur une diagonale allant d'arrière en haut et ouvert une longuc ct nette crevasse de 200 mètres cubes, toul en projetant le bourrage, et en laissant absolument nus lo puits ct la galcrie supérieure.

L'échec de l'essai de sautage ne laissait aux ingénieurs d'autre choix que l'ouvorture de la paroi par ctages, jusqu'au niveau de la galerie de prise d'eau.
Ce fut le parti que l'on adopta et l'on se mit au travail sous la menace des éboulements et des chutes de pierres qui mettaient constamment en danger la vie des ouvriers.

Le travail était poursuivi dans l'eau et la glace par des températures atteignant 20 degrés au dessous de zéro ; les opérations qui se terminèrent en $\mathbf{g}^{12}$, après cette terrible expérience de huil mois de travail répartis sur trois campagnes, ne pouvaient, on le conçoit, être exécutées que pendant quelques semaines, chaque année.

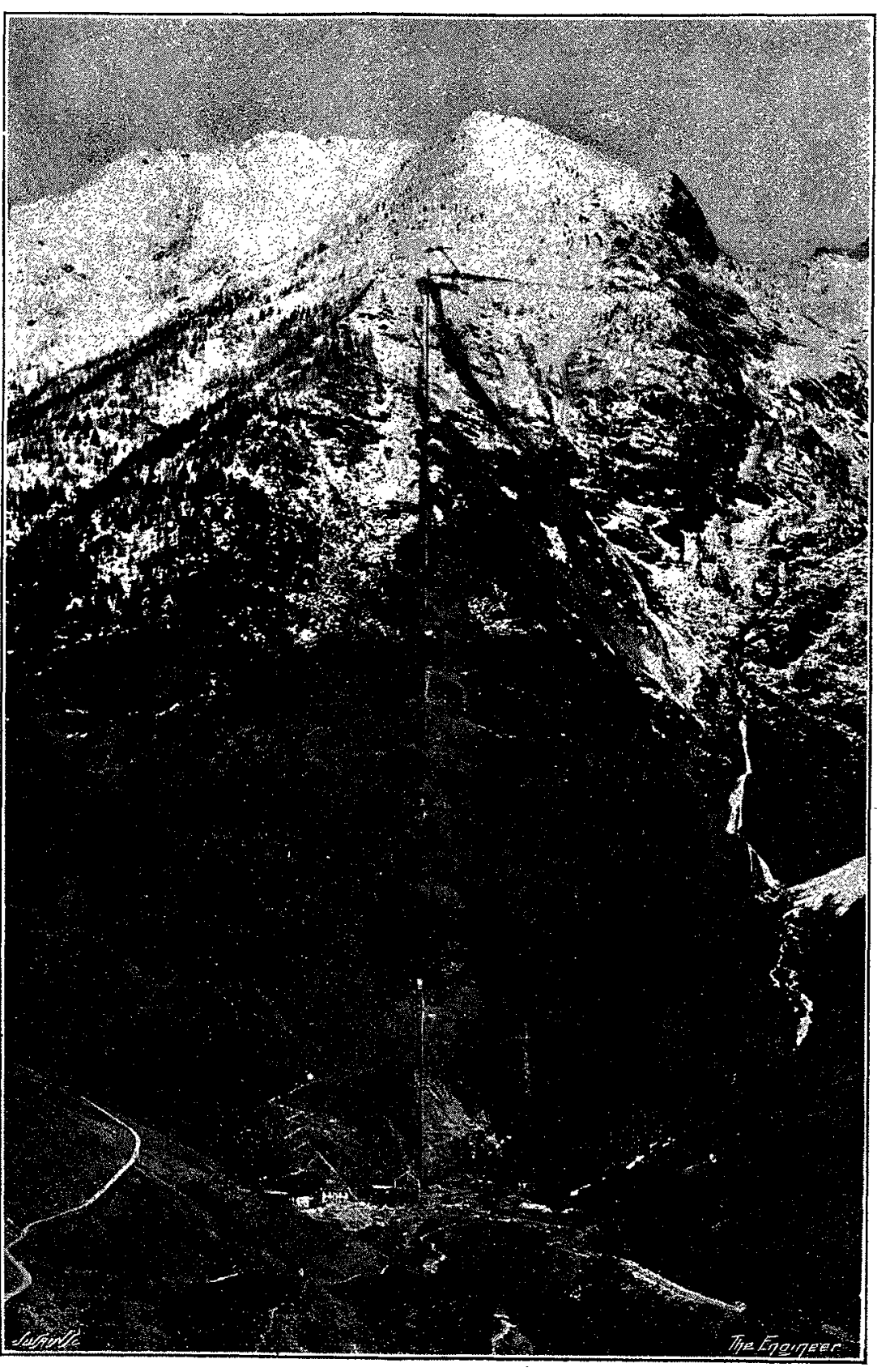

l'USINE D'ISOLA: ON VOTT LA CONDUITE SORTANT DE IJA MONTAGNE. A DROITE, LA POYA D'ARNO, EMISSAIRE DU LAC.
Pompes flottantes. - En attendant que la galerie de prise d'eau fût percée,l'installation des turbines et des machines étant achevéc ainsi que la pose des conduites forcées, il était indispensable de leur enroyer de l'eau. Pour cela et aussi pour faciliter l'ou verture de la tranchée dans la paroi du lac, on installa sur un bateau une baraque en bois abritant six groupes moto-centrifuges absorbant ensemble 6oo chevaux et débitant 3 ooo litres par seconde. Celte organisation coûta 20 ooo francs; elle. permit d'abaisser le nivea du lac assez bas pour qu'on pût poser en avant du puits $50 \mathrm{~m}$. de tuyaux de $\mathrm{I}^{\mathrm{m}} 50$ de diamètre formant lo commencement de la: galcrie. En tête de cette conduile on placa une grille destinice à cmpêcher l'entraînement des débris dans les turbines.

Un croquis fait par $M$. Vassena montre dans quel ordre successif les travaux furent cntrepris.

Les pompes resteront montées, elles formeront une partic permancnte dis l'ouvrage ; grâce à elles, on peut abaisser cncore de $7^{\mathrm{m}}$ au-dessous de la prise d'eau le niveau du lac, el c'est un volume supplémentaire de 2 ooo ooo de mètres cubes que l'on peut ainsi utiliser.

Déversoir. - Un premier mur avait été fait pour relever Je plan d'eau du lac. La limite de résistance qu'il offrait ayant paru trop faible, on construisit une nouvelle digue de section trapézoïdale ayant 20 mètres de largeur à la base et 3 mètres au sommet; elle est formée de blocs de r mètre cube et s'élève à 25 mètres au-dessus du niveau du lac.

Aménagement de l'Adamè supérieur. - Pour augmenter le volume d'eau reçu par le lac d'Alno, on a capté les caux de l'Adamè ; ces eaux arrivent par ùn tunncl de 6 kilomètres à r 80 mètres au-dessus du lac et alimentenont plus tard une troisième station devant utiliser l'énergic de l'eau avant qu'elle ne tombe dans le lac. 
Event de sécurité. - Pour garantir le tunnel en pression contre les effets de la houle sur le lac, on a foré près de son extrémité, à $9^{2}$ mètres en deçà de l'origine de la conduite forcée, une cheminée ou évent de $6 \mathrm{r}^{\mathrm{m}} 35$ de hauteur, dépassant de 5 mètres le niveau du lac. Cette cheminée est garnie dans sa partie inférieure d'une sorte d'entonnoir en tôle qui se raccorde avec la conduite métallique placćc dans le tunnel. Cette conduite, de $1^{\text {m}} 80$ de dianiètre, se termine par une pièce en forme de $\mathrm{V}$ dont l'un des hras est fermé par un joint plein.

Le second bras est relié par des vannes commandées électriquement aux 2 conduites forcécs actucllement montées.

CONDUITES roncées. -- Quoique la réalisalion de la chute supéricure en 2 élages n'eûl pas nécossité une dépense plus élevée a gu'elle cût permis de bónélicjer d'un grimd nombre d'exemples, I a Sociélé de l'Adamello, confiante dans les consinucleurs clialgés de la partic hydraulique de l'entreprise, accepta lours proposilions al so décida à uliliser l'eau d'un seul jet. I a longueur de la canalisalion, mesmécen projection horizontale, est de I $198 \mathrm{~m} .50$.

Le volum e d'eau à débiler

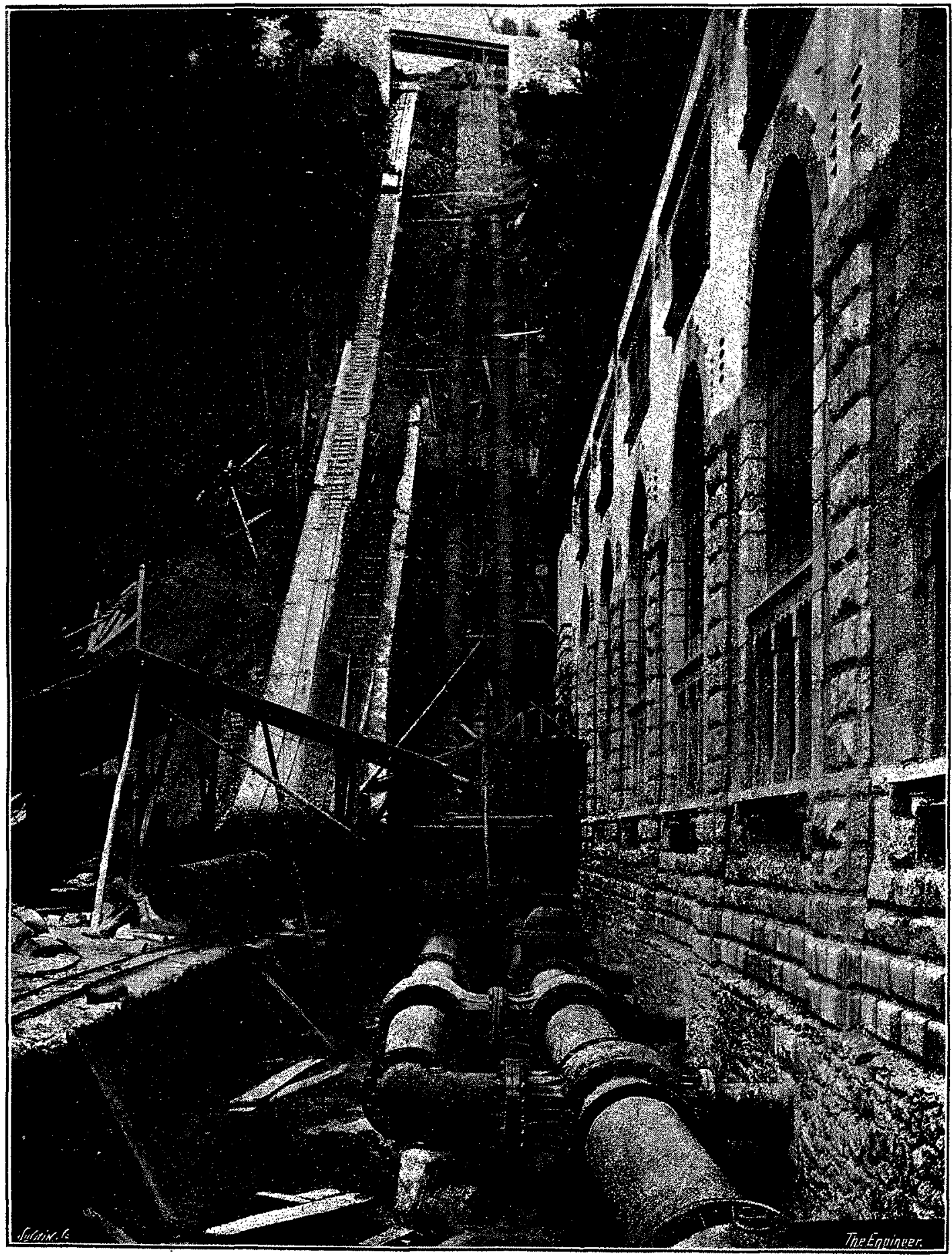

PAOTIE INFÉRIEURE DE IA CONDUITE RORCÉE ET TUBULUKE UE DISTRIBUTION DE LA STITION DE GEDEGOLO. $2^{\circ}$ section, tuyaux soudés, asscmblés à courre-joint ì double clouure; diamètre, $750 \mathrm{~mm}$. ; épaisseur 1 i à $17 \mathrm{~mm}$.; $3^{\circ}$ section, tuyaux de même construction que les précédents ; diamètre, 700 millimètres ; épaisseur, I 7 à $23 \mathrm{~mm}$.; $4^{\circ}$ section, tuyaux soudés, assemblés par brides avec insertions de joints de caoutchouc (on sait comment ils sont faits) ; diamètre, $650 \mathrm{~mm}$; ćpaisscur, 22 a 30 millimètres :

$5^{\mathrm{c}}$ section, mémes disposilions que pour la $4^{\circ}$ section ; diamètrc, $620 \mathrm{~m} / \mathrm{m}$. ; épaisseur, 29 à 32 millimètres.

Les tuyaux sont faits cn tôle d'a ci er Marlin, d'une résistance de 36 à $40 \mathrm{kgs}$ par millimètre carré, avec un cocfficient d'allongement de 30 pour roo. l.es longucurs des tuyaux étaient de 6 mères. I.es tryaux furent éprouvés à lalelicr à des pressions allant jusqu'ù i 35 almosphères, et, à la fin dé la consluclion, ì $g^{3}$ almosplic̀res.

I. a canalisalion est lenue par liwil ancrages. An-dessous de chacun d'rux sont placés des joints de dilalalion avec presseCloupes. Ella palse a lavers six tumbels percós dans la roche, le plus long a $150 \mathrm{nt}$; sur to reste do la longrvenr, elle est lankôt ral lanchéc, tamlôt à la surface dir sol.

Tuyaux de dis- et la vitesse, sous une pression de $9^{3}$ atmosphères, nécessitaient une conduite de grand diamètre et de très forte épaisseur. On préféra adopler une distribution au moyen de quatre conduites parallèles. On avait ainsi la possibilité de proportionner la dépense aux besoins immédiats.

Nature de la tuyauterie. - La longueur totale de la canalisation esl de $\mathrm{x} / 77^{\circ}$ mètres ; elle esl divisée en cinq sections : $\mathrm{I}^{\mathrm{ro}}$ section, partant de la galeric de prise d'eau, tuyaux rivés de 800 millimètres de diamètre, épaisseur croissante de 7 à 14 millimètres; tribution. - Les tuvaux ont 556 millimètres de diamètre et 32 millimètres d'épaisscur ; ils sont réunis de tolle façon que l'un quelconque d'entre cux peut alimenter loules les turbines de la station.

La voie funiculaire qui grimpait au flane de la montagne était à l'écartement culre rails de 50 centimètres; elle était placće entre la rampe préparćc pour les fulures conduites et les conduiles en cours de montage ; elle servit à transporter le personnel et les matériaux, un seul vagonnet assurant le va-et-vient. La voie avait r.6oo mètres de longueur, 
avec une pente maximum de rog̣ pour ıoo. Le câble, de 20 millimètres, en fil d'acier au creuset, d'une résistance garantie de $23 \mathrm{~kg}$. 5oo, était actionné par un treuil électrique de 70 chevaux.

Câbles aériens. - L'ouvrage le plus important de ce genre avait r.60o mètres de longueur et allait d'Isola au lac. Il comprenait un câble portcur de 22 millimètres pouvant supporter une charge de 750 kilogrammes et un câble tracteur de 5 millimètres, actionné par un moteur de 20 chevaux, donnant une vitesse de déplacement de $\mathrm{I}^{\mathrm{m}} 5 \mathrm{o}$ par seconde.

Blondin. - Ce type de porteur aérien fut employé pour un autre bâtiment de moindre dimension. On y a prévu l'installation de sept groupes, mais cinq seulement sont instullés. Le bâtiment contient, en outre, une excitatrice de 500 chevaux, actionnée par un moteur triphasé, et une excitatrice de 550 chevaux, coupléc avec une turbine tournant à 63 o tours par minute.

Les cinq turbines Pelton sont de 6500 chevaux chacune et ont été étudiées pour unc hauteur de chute variant de 853 à 930 mètres. Leur socle en fonte supporte la capote, les deux paliers, le régulateur et le pot d'échappement du régulatcur de pression.

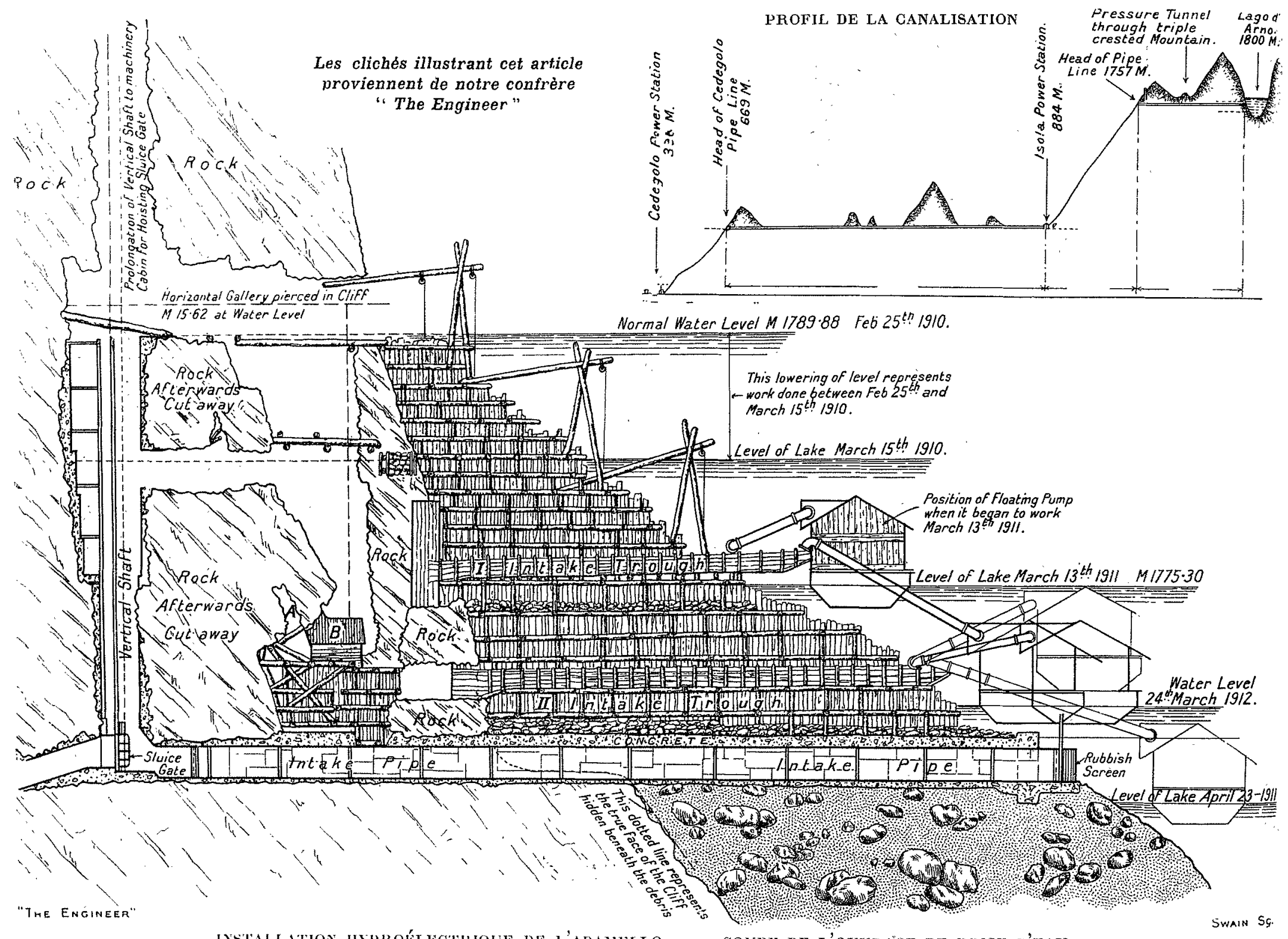

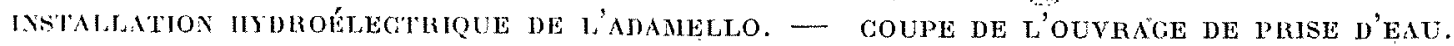

le lransport des bloes deslinés à la construction de la digue. formant la retenue du lec. Il comportait deux cables de 37 millimètres en acier, sur lesquels couraient des chariots portant des bennes ou des louves que l'on pouvait descendre au point voulu depuis la guérite de manoeuvre. Les moteurs des Ireuils actiommant les câbles de traction et de levage vaient de 95 chevaux, les charges maxima étaient de 3.500 kilogrammes el pouraicul etro déplacécs à la vïtesse de $\mathrm{I}_{20}$ par secoude. Un seul homme suffisail à la mancuvre des flifférents treuils.

\section{USINE D'ISOLA}

L'usine d'Isola consiste en un bâtinent principal de $63 \mathrm{~m}$.

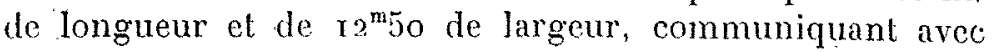

Lobjel principal visé dans l'étude de ces lurbines ful la parfaile régulation. On décida de donner à la roue mobile une forle janle, de façon que son action comme volant alté. nuât, dans une certaine mesure, les risques d'irrégularité, el l'on adopta la faible vilesse de 420 tours par minute.

Le diamètre extérieur de la roue est de 3 mètreś ct lo diamètre du jet est de 80 millimètres.

Les aubes, en acier fondu, sont polies comme des glaces "t leur bord est tranchant comme une lame de rasoir. Elles sont fixées à queue d'aronde et boulonnées sur la jantè du disque nobile.

La buse est du modèle à déviation. Elle est liée au régulatcur de telle façon que toute baisse subite de charge proroque un déplacement vertical de la veine, quii lui fait échàp- 
per les aubes et frapper le chéneau de fer qui conduit l'eau au canal de fuite. Pendant ce mouvement, l'aiguille piriforme en acier au nickel avance doucement, suivant l'axe de la buse, et règle le jet, tandis que la buse remonte à sa position normale.

Ce mode de réglage est supérieur au syslème par by-pass, ou au réglage par simple déviation, parce qu'il amoindrit lo coup de bélier tout en économisant l'eau.

Le régulateur à huile comprimée agit sur l'aiguille de réglage, pour les pelites variations, et sur la buse pour dévier le jet quand il est nécessaire d'arrêter brusquement la turbine. Ies essais de rendements faits en mai $19{ }^{2}$, en vue de

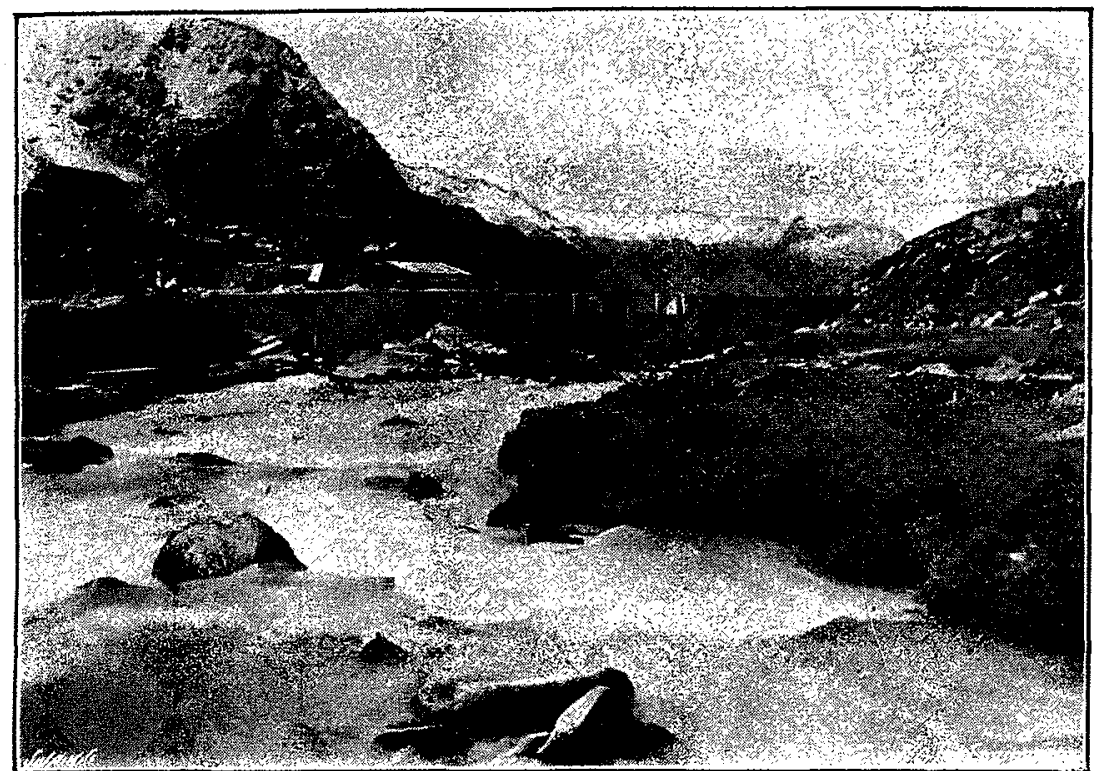

Irouve devant le réseau des canaux munis de rannes qui servent à la mise en charge de l'usine de Cedegolo.

Le canal de fuite des turbines d'Isola se décharge dans un canal de jaugeage par déversoir. Cclui-ci reçoit les eaux réunies des torrents Salarno et Adamè et, à quelques mètres de là, la Poglia d'Arno. A partir de ce point, les trois alfluents de la Poglia coulent dans un seul canal de ${ }^{\text {u }} 80$ de largeur, et forment une lame d'eau de $x^{\mathrm{m}} 60$ de hateur.

Le canal suit le flanc de la monlagne avec une pente de 2 pour roo et passe du versant de la Poglia dans celui de I'Oglio par un parcours de 4595 mètres, au milicu duquel on a ménagé 'un déversoir.

Le canal est construit particllement en maçonnerie de moellons el de ciment el parliellement en béton enduit de ciment. La paroi extérieure en a zo centimètres d'épaisseur, mais plus de la moilic de la longucur passe dans 5 tunnels.

Au sortir du dernicr, l'ea'u traverse nne grille el entre dans un bassin régulateur de mise en charge qui forme la têle de la conduite inféricure.

Cette conduite mesure $\mathrm{x}$ o $8^{\mathrm{m}} 85$ de longueur, of suivant la ha'uteur de l'ean dans le bassin régulateur, la différence de niveau entre la surface de la nappe et les buses des turbines varie de $467^{\mathrm{m}} 28$ à $47_{4} 4^{\mathrm{m}} 36$.

Le système de captage est ingénieux. Les torrents Adamè et Poglia d'Arno qui descendent du glacier d'Adamello, l'un tout contre le bâtiment de la station d'Isola et l'autre du côté opposé, sont barrés par de solides murs de $2 \mathrm{~m}$. de hauteur et de $2^{\mathrm{m}} 50$ d'épaisseur, reposant sur des fondations en béton de $\mathrm{I}$ mètre de profondeur, jetées à même les lits des torrents.

BASSIN DE MISE EN CHARGE SUR L'ADAM̀̀, VIDE.

l'allocation de la prime prévue aux constructeurs, montrèrent que les turbines donnaient un rendement de 80 p. roo à la charge minimum de 2000 chevaux, 88 p. 100 à la charge normale de 5500 chevaux, le rendement décrût un peu pour la charge maximum de 7500 chevaux. Le rendement avait une importance capitale; en effel, au prix de vente de 4 centimes le kilowatt-heure, chaque centième de rendement représente un gain annuel de plus de 28 ooo francs.

Le rendement fut établi en faisant débiter les généralrices triphasées sur des résistances d'eau et en mesurant les caractéristiques du courant. Un réservoir de 3 mètres de profonde'ur recevait l'eau des turbines et formait matelas; le volume d'eau débitée était automatiquement enregistré par un compteur placé sur le canal de fuite qui sert de prise d'eau à l'usine aval de Cedegolo.

Nous ne nous arrêterons pas à la description des machines électriques qui nous entraînerait trop loin et n'offre pas d'intérêt spécial dans l'aménagement de la chute.

Nous dirons seulement que les alternateurs manchonnés aux turbines donnent directement du courant triphasé à 42 périodes, sous une tension de to à 12000 volts, et que les excitatrices sont des machines à grande vitesse angulaire, à pôles auxiliaires et à collecteurs très longs et frettés par trois bagues d'acier.

\section{Instaliation de ta Station de Cedegolo}

En sortant de la salle des machines d'Isola, sur le terrain où sont les maisons des ingénieurs et des ouvriers, on se

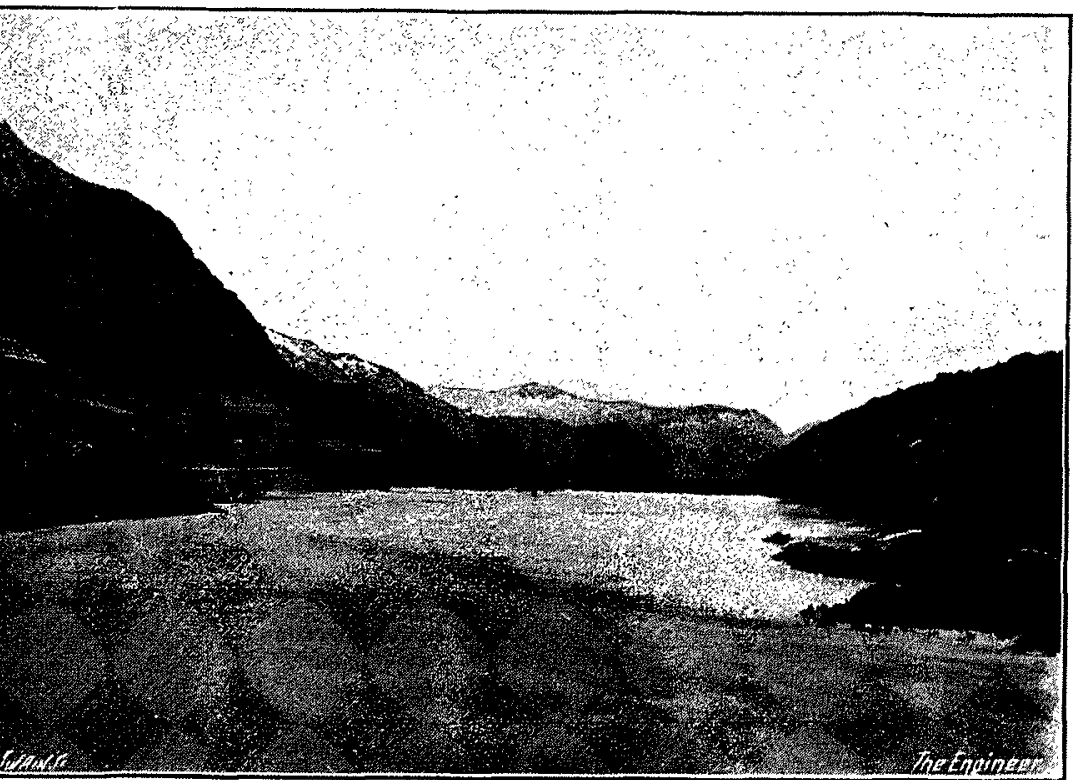

BASSIN DE MISE EN CHARgE SUR L'ADAMÈ, PLEIN.

Ils ont sur la rive gauche deux déversoirs que l'on règle, à la hauteur voulue, au moyen de madriers placés ou retirćs à la main.

Quant au Salarno; il fallut le capter au village de Frésine, à une altitude assez grande pour jouvoir le ramener à Isola. On dut lui faire rebrousser chemin parallelement à la Voglia dont il est un affluent.

La prise d'eau est formée par un harrage de $/ 4$ mètres de hauteur avec deux vannes de $\mathrm{x}^{\mathrm{m}} 20$ d'ouverlure sur. la rive gauche du torrent. L'eau est conduite dans un canal de béton enduit de ciment de r 563 mètres de longueur, dont 
plus de foo mètres en tunnel dans le rocher; le reste en est, en partie, couvert et en partic en tranchée ouverte. La pente du canal est de 1,5 à 2 pour 1oo. La largeur du canal est d'abord de $2^{\mathrm{m}} 25$, elle est ensuite rétrécie à $\mathrm{I}^{\mathrm{m}} 50$. la nappe d'eau a normalement $\mathrm{I}^{\mathrm{m}} 60$ d'épaisseur.

Le canal suit d'abord la rive gauche du Salarno, passe sous la route, débouche dans le bassin de la Poglia et, longeant la rive droite de celle-ci, coule dans une direction opposéc à la sienne, tantôt en tunnel, tantôt à découvert, presque jusqu'au village d'Isola. A ce point, il traverse l'Adamè sur un pont, reçoit ensuite la dérivation de celui-ci traverse en tranchée couverte les champs d'Isola et devient ntilisée pour en stabiliser les murs sur leurs fondations.

Bâti comme il l'est sur le seul espace que l'on ait pu rencontrer, étroite marche naturelle sur la pente rapide de la montagne, tout décimètre perdu dans l'épaisseur des murs du côté de la valléc représentait une augmentation coûteuse de fouille du côté de la montagne, ou bien conduisait à la construction de murs de section trapézoïdale de hauteur et d'épaisséur à la base impraticables. Le sol rochcux, coupé de larges fissures, offrait encore d'autres difficultés.

On renonça à l'emploi de maçonnerie; M. Danioli, ingénieur à Milan, spécialiste de constructions en béton, étudia un réservoir en ciment armé, n'ayant qu'un léger mur extć-

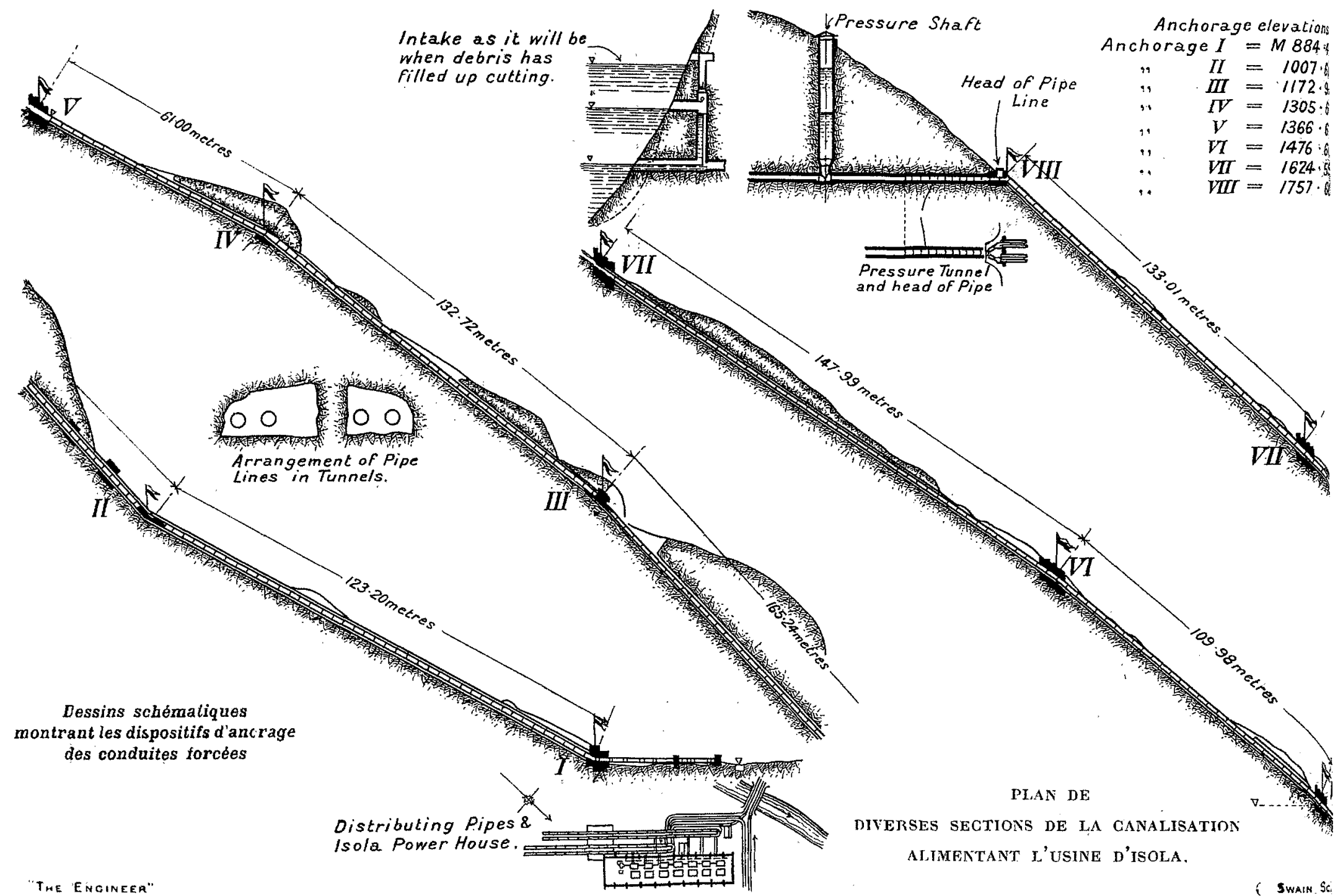

le canal de fuite de cette station; il reçoit alors l'eau provenant de la Poglia d'Arno et conduit toutes ces caux au bassin régulateur scrvant de mise en charge à la conduite de l'usine de Cedegolo.

Le canal aboulit à une chambre partagíe en deux par une cloison de béton armé de $3^{\text {m }}$ 'o de largeur, avec deux murs reliés par des tirants, munis de six ouvertures de a mètres de hautenr sur $\mathrm{I}^{\mathrm{m}}$ ?o de largeur, s'onvranl ì volonté sur $\mathrm{l} e$ réservoir compensateur ou sur la chambre de mise en charge, et capables, en outre, d'établir une communication entre eux.

Le réservoir compensateur, contenant près de 20000 mètres cubes d'eau, suffit pour alimenler les turbines de Cedegolo pendant deux heures, a raison de 3 joo litres par seconde en cas d'aceident motivant un arrêt ì la prise d'eau.

La construction de ce réservoir est extrèmement intéres$\therefore$ inte, la composante verticale de la pression statique ćtan! ricur de 40 centimètres d'épaisscur à la basc el $x 5$ centimètres d'épaisseur à la crête. Ge mur repose en partie sur la roche, en partic sur deux voùtes de 5 mètrcs d'ouverture et une troisième de 8 mèlres, jetées au-dessus des fissurcs.

On réalisa ainsi non seulement une économie dans la construction, mais encore un gain considérable de capacilt du réservoir dont la superficie est de 2 100 mètres carrés ; le fond, fait en brique, est incliné vers la vanne de la cloison qui sépare le réservoir de la chambre de mise en charge de. la conduile forcéc.

Une conduite spéciale permet de vider le réservoir pour le neltoyer ou y faire des réparations.

l'une des parois est formée par la montagne revêtue de maçonneric jusqu'à $?^{\text {"w }} 80$ de hauteur. Mu còté de la vallée, la paroi est formée par le mur léger en question. Mais ce mur ne supporte pas senl toute la charge, il ne constitnu 
cn effel que l'une dos parois d'un mur creux de construction très ingénieuse. A 4 mètres de distance du mur extéricur est un second mur semblable, en béton armé, relié au premier par des cloisons verticales et des cloisons horizontales qui forment des alvéoles dans lesquelles l'eau du réservoị pénètr'e par des ouvertures ménagées dans le mur intérieur. Ces alvéoles mesurent $4 \mathrm{~m} . \times 3 \mathrm{~m} . \times 2^{\mathrm{m}} 35$, elles sont dispo- et sont rivées, les suivantes ont 13 ì 26 millimètres ch sont soudées au gaz à l'eau.

Les joints de dilatation sont à presse-étoupes avec girnitures de chanvre suiffées, ils sont calculés pour une diff́́rence de 40 degrés entre la lempérature minima el celle du moment où les tuyaux ont été mis en place.

Onze trous d'hommes permeltent de visiter chaque conduite. Une vanne placéc au bas des conduites avant le tuyau de distribution permel. de les vider sans avoir à en faire passor l'cau par les turbines.

Les conduites sont placées, sur loule lew longueur, dans une tranchée laillée ditls: le roc; elles sont absolument reclilignes en projection; elles reposent sur des socles en fonle et présentent troize points de changement de pente. A chacun d'cux, on a lail un ancrage au moyen de tirants scelles dans la maçonnerie.

La roie à traction funiculaire qui a servi au lransport des tuyaux a 50 conlimètr's d'écartement comme celle d'lsola au lac: d'Arno, mais à l'inverse de celle-ci ell' fonclionne avec deux vagomnels s'équilibrant et a un croiscment en double voic an son milieu. Elle a $\mathrm{s} 056^{\mathrm{m}} 26$ de longueur, la différence d'allitude entre ses deux cxlr'imités est de $926^{\mathrm{m}} 66$; ce qui donne unc pente moyenne de 52 pour roo, mais $l_{1: 1}$ pente maxima atteint $9^{3}$ pour roo.

Le câble d'acier aux extrémilés duqucl sont accrochés les vagonnets a 25 millimètres de diamètre, sa résistance à la ruplure

VUE D'UNE TURBINE PELTON DE 6500 CHEVAUX.

sées en trois rangs, et leur capacité totale est de près de 4 ooo mètres cubes, qui vient augmentex d'autant le volume du bassin et dont le poids pèse sur les murs et augmente leur stabilité sur leur fondation, dont la profondeur n'est que de $65 \mathrm{~cm}$. IJe développement de ces murs a $130 \mathrm{~m}$. et leur conslruction ful cxéculéc en cent journées environ.

La chambre de mise en charge, dans laquelle peut clre vidé le bassin compensaleur, a rom85 de longueur, $5{ }^{\mathrm{m}} 25$ de largeur et ro mètres de profondeur. Le radier en bélon repose sur une fondation rocheuse. Le mur exlérieur a $5^{m} 50$ à la base el 2 mèlres d'épaisscur à la crête.

Dans le bas du mur sonl scellees les premières longueurs des trois conduites forcées. L'entrée des combuiles est garnie de grilles.

Deux conduites seulement ont été montécs, la lête. de la troisième est fermée extéricurcment par un joint plein.

Les deux conduites mises en place sont munies de reniflards de $50 \mathrm{~cm}$. de diamètre et ro $\mathrm{m}$. de hauteur, dépassant de $x$ mètre la hauteur maximum de l'cau dans la chambre.

Les tuyaux sont divisés en trois sections d'environ $300 \mathrm{~m}$. chacune et ont $\mathrm{x}$ 030, goo et 835 millimètres de diamèlre. Ils sont faits en tôle d'acier Martin pott chaudières, d'unc résistance de 38 à 40 kilogrammes par millimètre carré et d'un coefficient d'allongement de 24 pour cent. Les viroles des premières sections ont de 5 à I 6 millimètres d'épaisseur

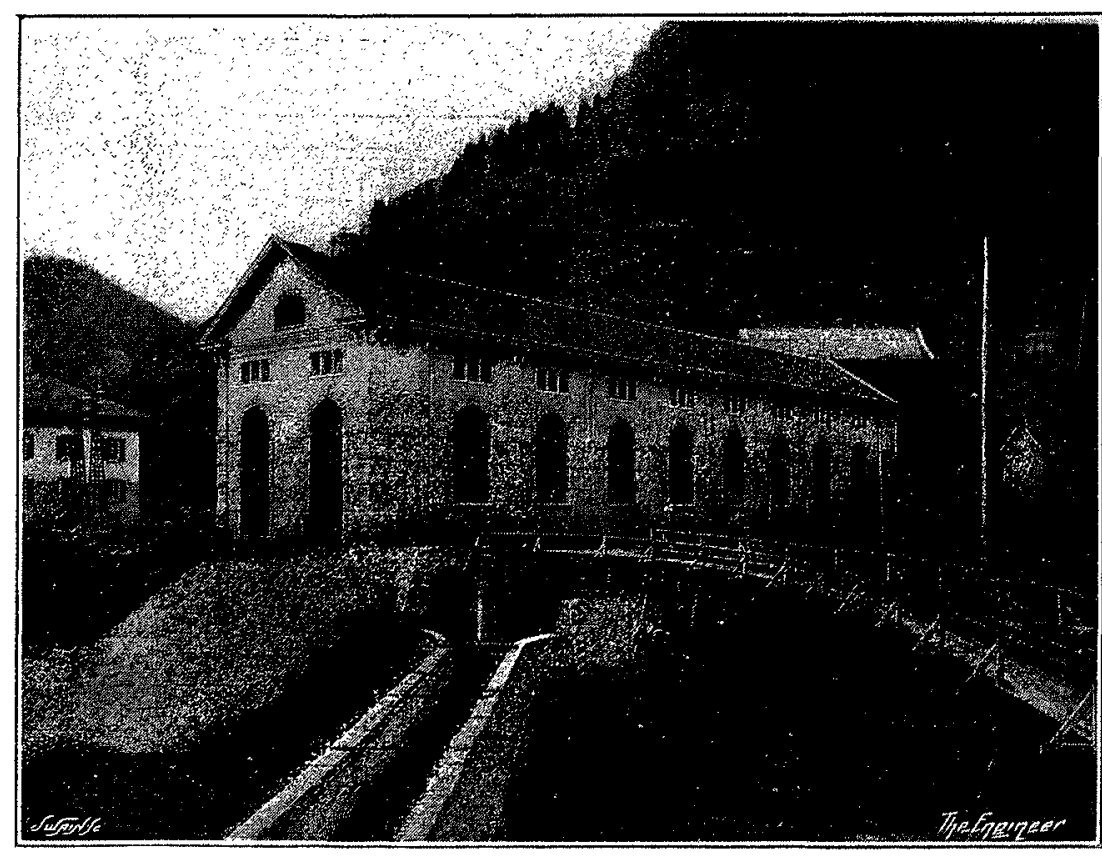

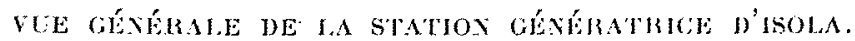

est de 7500 kilogrammes, mais on ne l'a jamais soumis à une charge excédant 5 ooo kilogrammes.

Un moteur électrique de 60 chevaux fait fonclionner la câble et lui imprime une vitesse en rapport avec la charge des vagonnets. La machinerie comporte deux freins actionnés soit à la main, soit par un électro-aimant.

Les conduites de distribution ont $38^{\mathrm{m}} 85$ de longucur, elles forment le prolongement des deux conduites forcéres actuel- 
lement en scrvice. Elles sont placées parallèlement à la salle des machines et à I mètre en contre-bas du sol de celle-ci, dans une tranchée de $6^{\mathrm{m}} 6 \mathrm{o}$ de largeur garnie de béton. On y a ménagé la place d'une troisième conduite qui fera suite à la troisième colonne à mettre plus tard.

Ces conduites de distribution de 800 millimètres de diamètre el 26 millimètres d'épaisseur sont divisées en tronçons assemblés par des joints à brides et boulons avec insertion d'anneaux de caoulchouc.

Elles alimentent, comme on l'a dit, cincl turbines, au moyen de tuyaux en acier soudé de $\$ 50$ millimètres de diamètre. Un sixième tuyau de 250 millimètres actionne la lurbine d'une des excitatrices et reçoit à voJonté l'eau de l'ume ou de J'antre colonne. - I ces vannes de ces diverses lurbines sont munies de servo-moteurs hydrauliques.

Les I too mètres de tuyaux de chacune de ces conduites forcées, qui croisent sur leur parcours quatre chemins vicinaux et deux sentiers, ont élé posés, y compris les tuyaux de distribulion, en moins de six mois. Le travail fut attaqué en quatre points à la fois. Une première épreuve ful faite en mettant les conduites en charge avec $200^{\mathrm{m}}$ do haulcur d'eau, de façon à Jaisser aux maçonneries des ancrages le lemps do faire prise, puis on les soumit aux essais hydrauliques habituels.
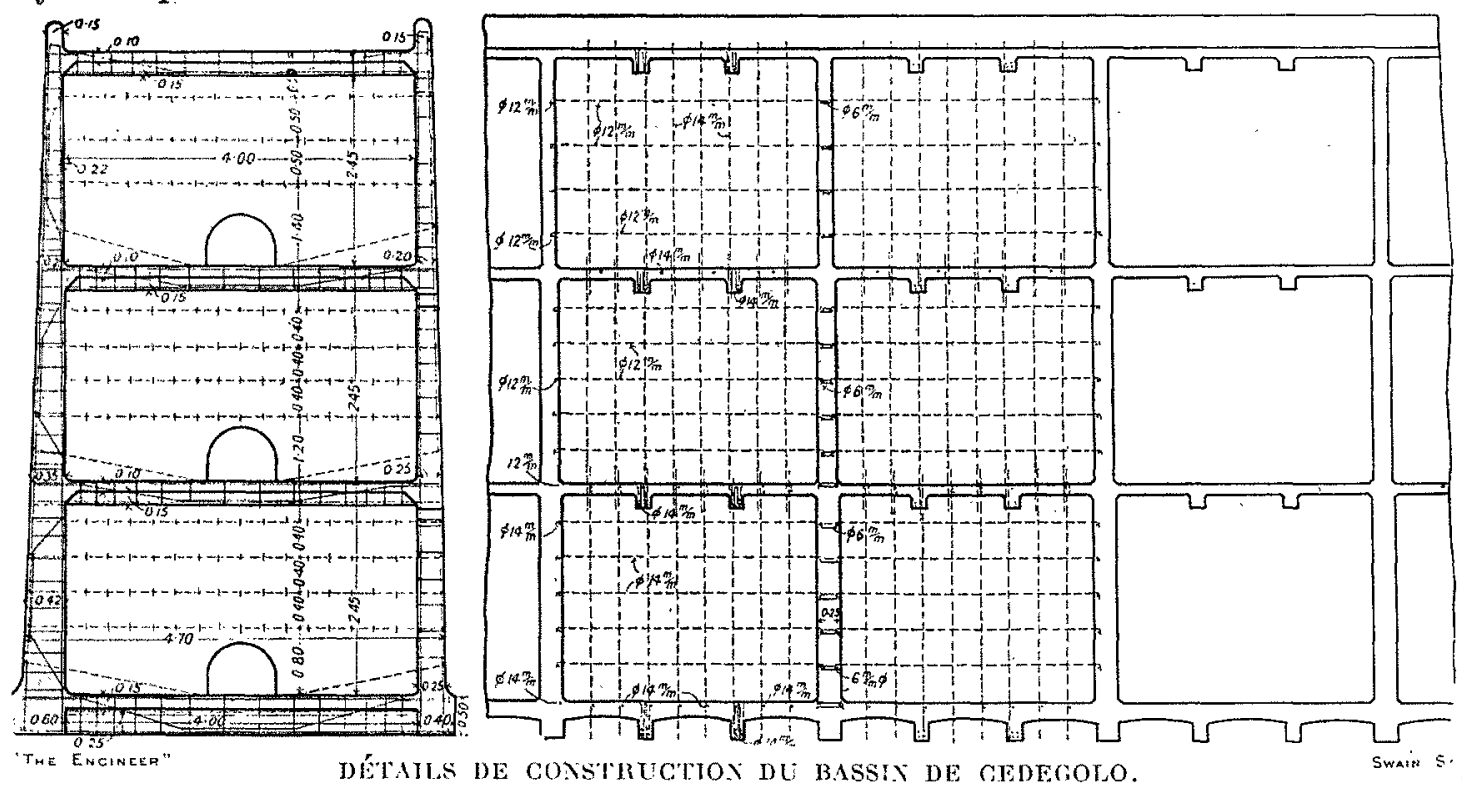

appropriée. En le prenant pour base, la Société Générale de l'Adamello dispose d'un total annuel de 130 millions de kilowatt-heures dont elle utilise à présent 80 millions.

Il y a moins de quatorze ans que la station de Paderno lançait pour la première fois du courant sous $\times 4$ ooo volts sur une ligne de 33 kilomètres. Sa mise en marche marqua une époque et ouvrit une voie. Grandes installations, haules tensions, transmission à longue distance, tels apparurent les principes des programmes futurs.

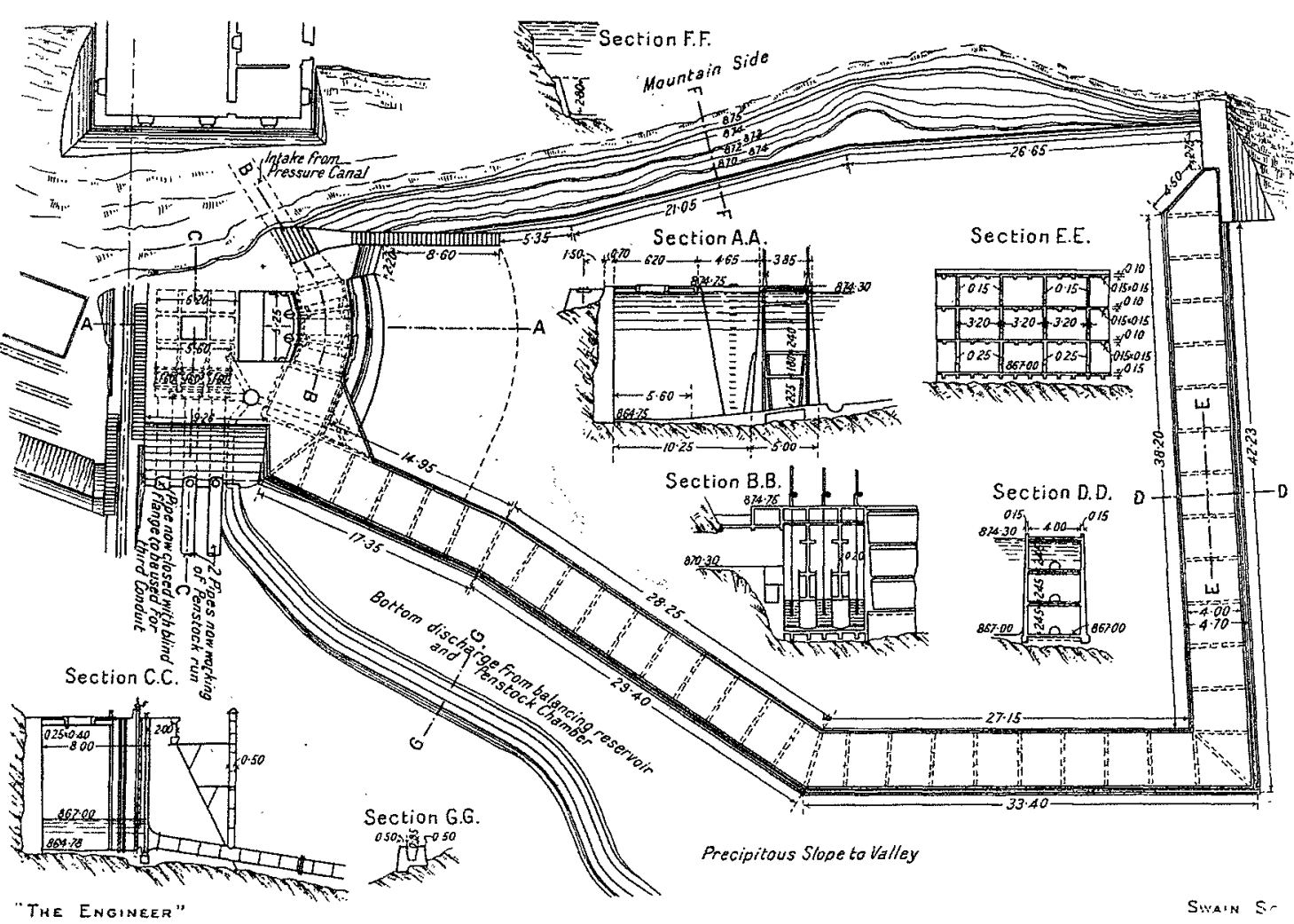

PLAN ET DÉTAILS DU BASSIN DE MISE EN GIARCE DE LUSINE DE GEGOLO. de Paderno eut réduil à néant les objections des timorés, la Société Conti transportait du courant à r20 Kil. sous 56 ooo V. ; l'année suivante (rgro), l'Adamello envoyait à yn5 Kil. du courant sous une lension de $60000 \mathrm{~V}$., portée ensuite à $7^{2}$ ooo. La Compagnic Terni-Rome emploie du courant à $7^{5}$ ooo volts et la station de la Pascara enveria à la lin de celte année du courant sous 88 ooo volts à Naples, situé à I 80 kilomètres.

De toutcs ces Sociétés, celle de l'Adamello offre probablement té plus d'intérêt au point de vuc techinique on raison des grandes difficultés qu'on a dû surmonter pour doter cette installation d'une

Nous n'entreprendrons pas non plus de décrire en détail la partic mécanique et électrique des stations d'Isola, de Cedegolo, el des sous-stations qui reçoivent le courant à $7^{2}$. ooo rolts el le ramenent à un voltage en permettant la distribulion el l'utilisation. On se bornera à donner une idée de la puissance de l'installation dont il vient d'ètre question.

L'évalualion en chevaux ne peut exprimer correctement la puissance d'une source d'énergie d'un débit irrégulier. Le kilowatt-heure est dans ce cas une unité de mesure plus réserve d'eau la rendant indépendante d'une station thermique. La puissance pourra être augmentée dans une largé proportion, quand les torrents, dont la Société a obtenu la concession scront tous aménagés. On peut la citer comme un modèle d'usine hydraulique parmi celles qui distribuent l'électricité en Italie.

La chaîne des Alpes, il est vrai, a deux versants. Mais le souci de rendre justice à l'énergie, à l'audace et à la ténacité des ingénieurs italiens ne saurait nous faire oublier que c'est 
de ce còté des Alpes que M. Arislide Bergès, en 1869 , ¿ I.ancey, M. Charles Chevrant, en r 888 , à Revel, el M. Bravel, en $18 g h$, à Chapareillan, onl fait les permières installations de hautes chutes.

Après ces pionniers, admirablement sccondés et aidés par les constructeurs de Grenoble, les Bouchayer et Viallet, les Joya, les Brenier-Neyret, les Bouvier, clc..., la voie était tracée, les principes posés et les éléments de calcul appuyés sur des faits écartant toute incertitude.

Nous pouvons applaudir aux succès des ingénieurs qui ont aménagé les chutes de l'Adamello, parce que nous pouvons constaler avec satisfaction que les ingénieurs et les conslrucleurs francais, en réalisant la chute d'Orlu $(936 \mathrm{~m}$.) dans l'Ariège et celle, presque verticale, de la Corbière $(736 \mathrm{~m}$.) en Savoie, ne se montrent point inférieurs en science, en aludace et en habileté à leurs confrères transalpins.

\section{LA NAVIGATION INTÉRIEURE EN SUISSE}

Notro excollent confrère le Bulletin technique de la Suisso. Romande publie une intéressanle élude de M. A. ParisWrTrec sur les grands projets de navigation intérieure qui sont acluellement discutés en Suisse. Nous en exlrayons Jes renscignemenls suivants

Le Conseil des Etats suisses est saisi du projet de législalion fédérale sur l'utilisation des forces hydrauliques, qui passera ensuile devant le Conseil national. Les Chambres vont donc prendre des dispositions lourdes de conséquences sur l'avenir de la navigalion sur les rivières suisses.

Nous avons signalé les grands travaux qui s'exéculent dans la Suisse romande, en particulicr le canal d'Fntreroche, qui cst l'amorce de la grande voie navigable du Rhône au Rhin en pussant par les lacs jurassiens, l'Aar et le Rhin de Bâle a Constance. C'est cetle dernière partie de cette voie que les nouveaux projots de loi intéressent tout particulièrement

L'éludo de l'aménagement dos forces motrices du Rhin est cnlrée dans tune phase décisise. Tout récemment on a achevé la construction des usines d'Augst-Wylen, dont le barrage relicnt une hauteur' d'eau de $8 \mathrm{~m}$. environ et forme en amont un lac de $7 \mathrm{~km}$. de long. Le capital investi de $22 \mathrm{mil}$ lions en fait un ouvrage définitif. Et co n'est que grâce aux ffforts personnels des partisans de la voje d'eau que l'éclusc d'Augs a été conslruite en vue de la grande navigation. Le projet portait une écluse pour petits chalands et flottage, donl le coût eût été de 450 ooo francs. La grande écluse n’a pu êlre construite qu'ensuite d'apport de la différence de loo ooo francs, moitié par la Suisse, et moilié par le Grand Duché de Bade. Il est inadmissible que la Société, qui va s'enrichir en exploitant le bien commun, se soit fait payer près d'un demi-million pour respecter la navigabilité actuelle en ce point du Rhin. Le fait est d'autant plus singulier que la navigation internationale sur ce point était garantie par les traités (Vienno I8I5 ét traité entre la Suisse et le Grand Duché de Bade 1879 ). Sans l'énergie de l'ingénieur Gelpke, de Bâle, en particulier, c'est un million qu'il cût fallu débourser d'ici quelques années peut-être pour rendre au Rhin sa navigabilité pour les grands chalands.

L'expérience est malheureusement faite actuellement à Laufenburg. L'écluse insuffisante devra inévitablement être prolongée à grands frais plus tard, aux dépens du pays, el cẹci pour remédier aux économies d'une société montée au copital de 40 millions. Et comme il y a en ce moment cing grands barrages sur le point de se construire sur le Rhin, la Suisse se demande avec inquićtude quelle atlitude la (anfédćration prend vis-à-vis des socićtés auxquelles elle siupprête à donner des concessions désormats. Fst-elle arméc pour exiger le respect du bien public ? Le sera-t-elle apris lo vote de la loi en discussion? Rien n'est moins certain. En effet, l'article 6 intitulé " Protection de la navigation en général " dil que "les usines hydrauliques scront construites de façon à ne pas entraver la navigation dans la mesurc oì elle cxiste n. Or, le fleuve, dans l'état actuel, avec ses rapide; ne permet pas la grande navigation intérieure, mais mo fois ces rapides apaisés el sa beauté, patrimoine national, en partie perdue, le fleuve sera de fait une série do lacs, el il serail facile de les mettro en communication suffisante cntro cux pour permetlre à la grande navigation existant en aval de profiter de ees nouvelles circonstances pour se prolonger en amont; el pénétrer au cour de la Suisse. Celle légitime compensation à la concession serail juste el admise sans difficullé par les requérants avanl la signature du contral. L'Autorité devrait seulement avoir te droit d'en dentander. l'exécution gratuile. Ce droil, c'est à la loi en discussion do l'octroyer sans limitation au pouvoir central, conseillé jart des techniciens experts. Si les Chambres n'y veillenl, a Suisse se trouvera dans quelques annces coupéc de la mor par des écluses insuffisantes, el obligée de l'ansborder los grands chalands sur des bateaux proportionnés à ses petiles installations. A moins loutefois qu'dle ne préfere, à chaqua nouvelle construction, répéler lo paicment des indemnilí: réclamées par la Sociélé d'Augst, pour exéculer après signa t'ure du contral, ce gu'clle aurail fail gratis si on l'avail de mandé à temps. Il s'agissait pour elle d'une augmentalion de moins de deux pour cent de son capilal de premier char blissement. Les dividendes cux-mêmes ne s'en seraient pas ressentis.

La navigation inlérieure sur chalands suisses nculres peut seule la garantir contre l'embargo on cas de guer" européenne. On se représente la diselte g'ui s'en suivrail, of ce que deviendrait son indépendance lorsque loules ses from. tières seraicnt fermées, el que les approvisionnemonts se raient impossibles. La navigation internationale, neutralise" par les traités, est la vraje solution suisse de la question do: farines en particulier. Et le moyen de la methe sur pied i bref délai est bien de lui assurer d'ores et déjà les outils dont elle a besoin, sans lui occasionner par la suite des fraits qui meltraient sa justification financière en question.

Gette justification ne saurait en effet tarder. La premic̀r année d'exploitation, xgoo, indiquait un trafic Strasbourg. Bâle de 300 lonnes. La dixième année, rgog, on notait 4o8 89 , et l'année courante, $\mathrm{Ig}_{\mathrm{I}} 3$, indique un apport à Bâle supérieur à 100000 tonnes, soil $\mathbf{1} 50$ pour soo de plus qu'il y a quatre ans. Une fois la régularisation du lac de Constanci assurée, ce trafic, qui se borne à 200 jours en aval de Bâle, sera assuré pour la majeure partie de l'année, si ce n'est pour l'annéc entière. Ce grand centre commercial scla alors en communication à peu près constante avec l'Océan, ol l'on ne verra plus se reproduire des arrêts prolongés des approvisionnements en Allemagne, comme le fail s'est passé au cours de la crise balkanique, où x 500 wagons de blé, achetés par la Confédération, fur»nt retenus deux mois aux entrepôts de Mannheim, sous prétexte de manque de mirtériel roulant. Cette masse énorme de farine n'aturait-elle pas été peut-être la bienvenue pour l'armée allemande en cas de conflit occidental européen ?

Notre confrère demande avec raison que l'article visé plus haut soit modifié et remplacé par la rédaction suivante : 